\title{
Blue-LED Calibration on DOAS System to Measure Nitrogen Dioxide Emission Compared with USEPA Method 7B Standard
}

\author{
Januar Arif Fatkhurrahman and Ikha Rasti Julia Sari \\ Balai Besar Teknologi Pencegahan Pencemaran Industri (BBTPPI) \\ Jl. Ki Mangunsarkoro No.6 Semarang, Telp. (024) 8310216, 8316315 \\ Email : januarfa@kemenperin.go.id
}

\begin{abstract}
Air polution level measurement sometime needs tricky instrumentation, costly, and use chemical reagent that could bad impact to environment, it also time consuming for analysis. In other hand, air polution level measurement, include nitrogen dioxide (NO2) needs accuracy, rapid and environment friendly for its analysis and measurement. Differential Optical Absorption Spectroscopy (DOAS) develop as spectrum measurements both UV and visible, transmitted by specific canal using absorption Lambert Beer's Law principal. On this basic method, NO2 measurement needs light source with $330-500 \mathrm{~nm}$ wavelength, it is possible to use cheap blueLED for this measurement. This research intends to calibrate prototype instrumentation for measuring NO2 by DOAS based using Blue-LED $(375 \mathrm{~nm})$ passed in continual gas container. $\mathrm{NO} 2$ emission simulated in laboratory scale by reacting copper $(\mathrm{Cu})$ with nitric acid $(\mathrm{HNO} 3)$ result NO2 gas formation. Blue-LED spectrum analysed by compact CCD Spectrometer for its absorbance spectrum, then calibrated with NO2 measurement using USEPA Method 7B standard that is commonly used in testing laboratory as standard method for NO2 measurement. It has good corelation between spectrum absorbance in CCD Spectrometer to USEPA Method 7B with more than $95 \%$ linierity. As rapidity of this measurement and shown good accuracy, more development for this method could carry fast, accurate, cheap, also environmentally friendly method for NO2 measurement.
\end{abstract}

Keywords: DOAS, Blue-LED, USEPA Method 7B, CCD Spectrometer

\section{Introduction}

Nitrogen Dioxide commonly major pollutan in industrial emission gas that give several bad effect for environment, both for worker health aspect also for decreasing environmental quality, including childhood asthma (Naidoo 2019). In industry nitrogen dioxide released from utility process like boiler, heater and generator set, while others generated both from anthropogenic, biomass burning, and soil emissions (R. Zhang, Tie, and Bond 2003). For environmental conformity industry periodically inspect their NO2 emission using environmental testing laboratory. They used standard method like SNI, the Indonesia Standard, JIS, the japanese standard, or USEPA, the US Standard. This measurement method is accurate, but seems resulting bad effect for environment as this method using chemical reagent that need another waste treatment (L. Zhang et al. 2018)(Ivanković and Hrenović 2010), also industry could take long time to get this result from testing laboratory. Several alternative method developed to resolved this problem, industry could use electrochemical based sensor (Cross et al. 2017) and chemiluminescent based instrument (Maeda, Aoki, and Munemori 1980) for NO2 measurement so they can get the result in realtime. But this method seems unreliable for lower and middle scale industry, as they dont have enough extra cost for environmental testing. (Platt and Stutz n.d.) nowadays, differential optical absorption spectroscopy (DOAS) seems to be another breakthrough for gas measurement, DOAS widely used in investigating stratospheric and troposheric air quality (Otten et al. 1998). Concentration of the gases retrieved from their absorption characteristic in the UV-VIS spectral range, originally this method based on Lambert-Beer law (Stutz et al. 2017). As DOAS spectrum quantification needs to be calibrated to inform true quantitive value, calibration using comparative method with USEPA Method 7B Standard will be additional 
breakthrough for another step research in DOAS method (Zielcke et al. 2013). For this challenge, we evaluate in comparative method between DOAS and USEPA Method 7B for NO2 emission measurement, also formulation for future work for DOAS emission measurement system made in latest technology like Integration of IoT technology

\section{Experimental Details}

Main idea of this research is developing NO2 gas measurement system on DOAS based system. In this research, NO2 generated by reacting Copper metal with diluted Nitric Acid, this reaction generated NO2 gas follow this reaction path (Jörg Kleffmann, Thorsten Benter, and Wiesen 2004):

$\mathrm{Cu}(\mathrm{s})+4 \mathrm{HNO} 3(\mathrm{aq}) \rightarrow \mathrm{Cu}(\mathrm{NO} 3) 2(\mathrm{aq})+2 \mathrm{NO} 2(\mathrm{~g})+2 \mathrm{H} 2 \mathrm{O}(\mathrm{l})$

We variate $0.01 \mathrm{~g}$ and $0.03 \mathrm{~g}$ copper metal reacted with fixed nitric acid molarity $(3 \mathrm{M})$, also we use $0.01 \mathrm{~g}$ copper weight in nitric acid variation $(2 \mathrm{M}-9 \mathrm{M})$. Generated NO2 gas flowed into the measuring path with $15 \mathrm{~cm}$ path length, made from acrylic tube. One of its end, we setup $415 \mathrm{~nm}$ single wavelength LED (lieteratur). Another end connected with optical fiber that directly pointed to CCD Spectrometer, we use Thorlabs CCS 100 Spectrometer in this research. CCD Spectrometer analyse intensity absorption varians for each generated NO2. This intensity varians analyzed with Excel. Typical blank intensity and NO2 concentration intensity observed like figure 1.

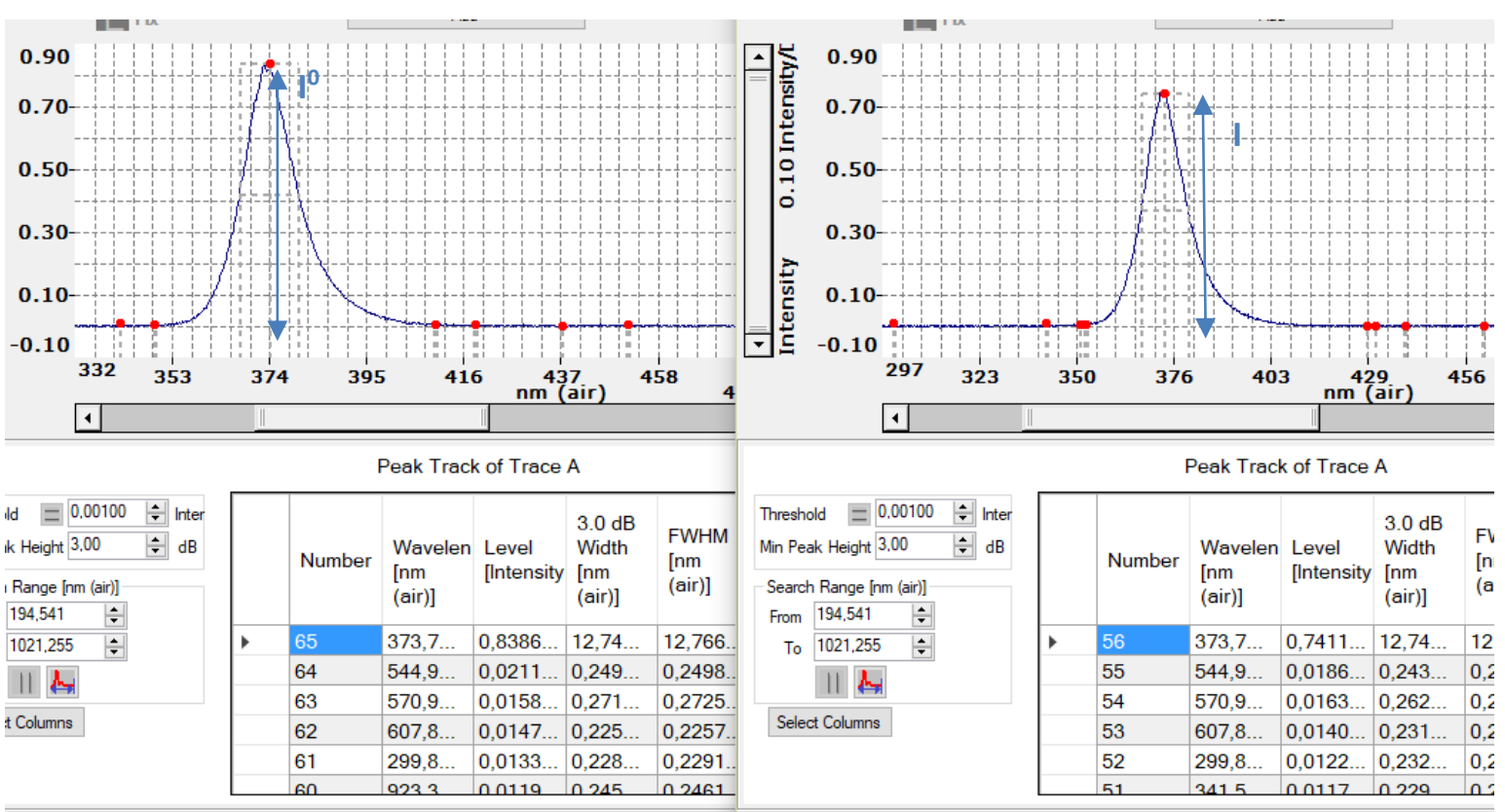

Figure 1. Typical Absorption Intensity on CCD Spectrometer; (left) blank intensity, (right) NO2 concentration intensity

NO2 gas outlet then connected to vacuum tube with USEPA Method 7B reagent, it will be analyzed by spectrophotometer to get $\mathrm{NO} 2$ concentration, later this method we used as quantification reference and linearity test. All of the experimental setup visualized as figure 2. 


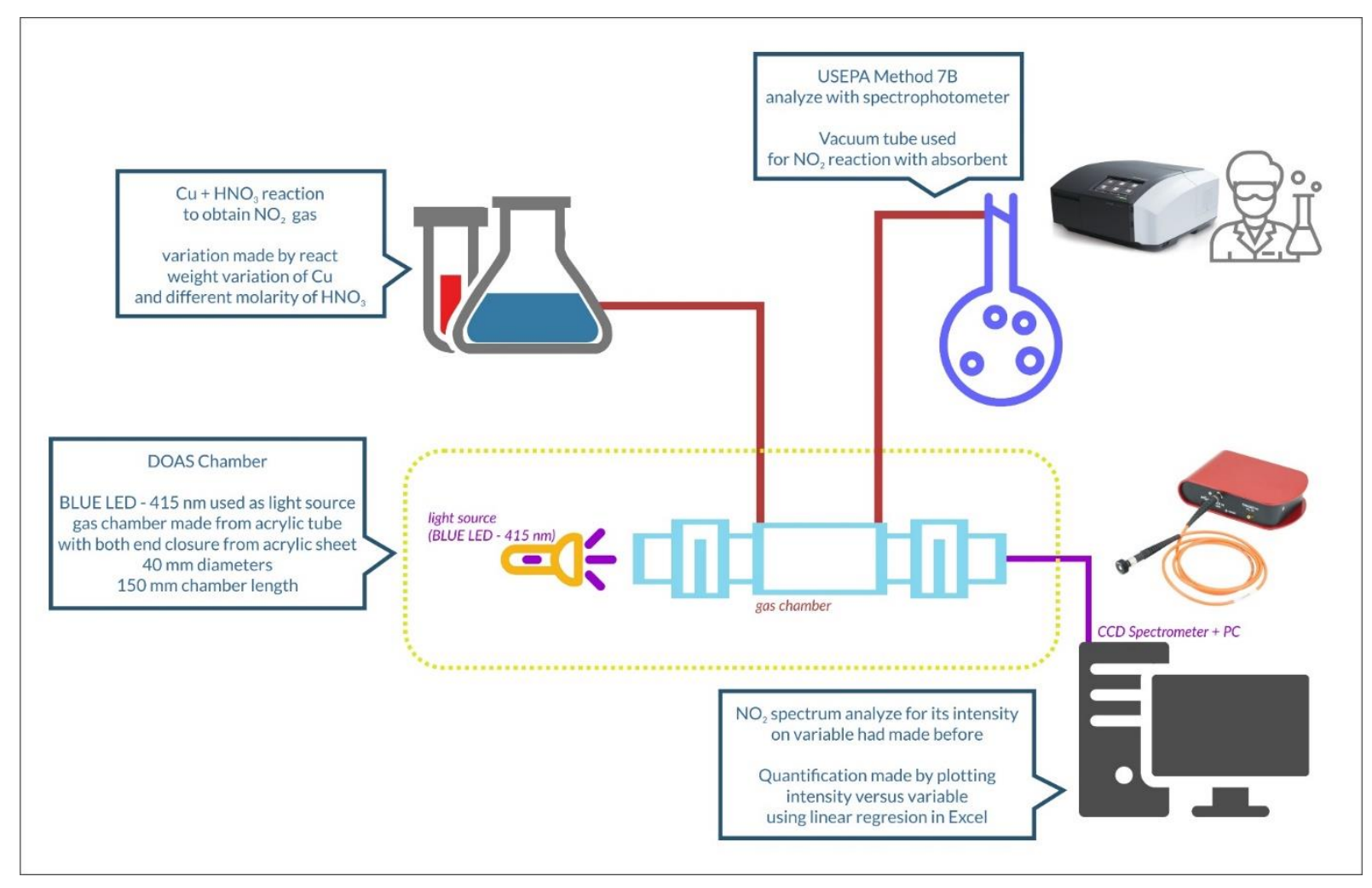

Figure 2. Instrument Setup for Measurement

\section{Result and Discussion}

From the experiment, we get NO2 spectrum absorbance for each variables. This NO2 spectrum absorbance plotted with $\mathrm{NO} 2$ concentration from spectrophotometer analysis based on USEPA method 7B like visualized on figure 3. These two variables may generate NO2 concentration from $20 \mathrm{mg} / \mathrm{Nm} 3$ to $1500 \mathrm{mg} / \mathrm{Nm} 3$. This experiment conduct by two variations of Copper weight, $0.01 \mathrm{~g}$ and $0.03 \mathrm{~g}$ with different nitric acid molarity.

Absorbance Correlation to NO2 Concentration (Cu $0.01 \mathrm{gr}$ )

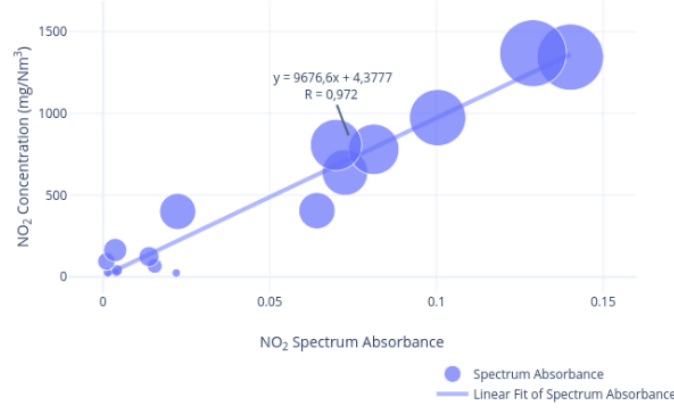

Absorbance Correlation to NO2 Concentration (Cu $0.03 \mathrm{gr}$ )

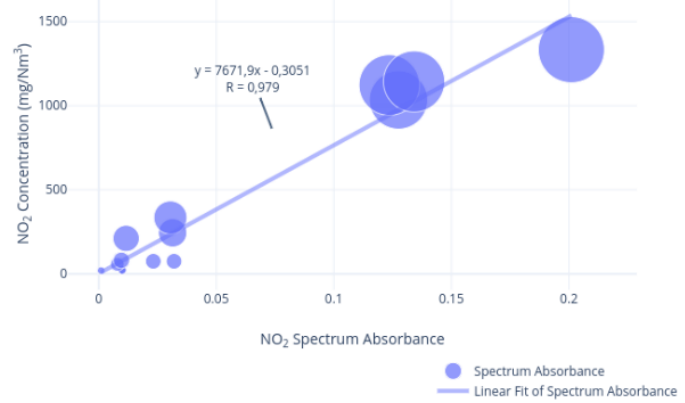

Figure 3. Absorbance Corelation (a) $\mathrm{Cu} 0.01 \mathrm{~g}$ and (b) $\mathrm{Cu} 0.03 \mathrm{~g}$

Copper weight correlation to NO2 concentration for both variable reach more than $95 \%$. Also resulting identical respond from two variables. This indicate positive correlation between NO2 spectrum absorbance to NO2 concentration quantified with USEPA Method 7B. 


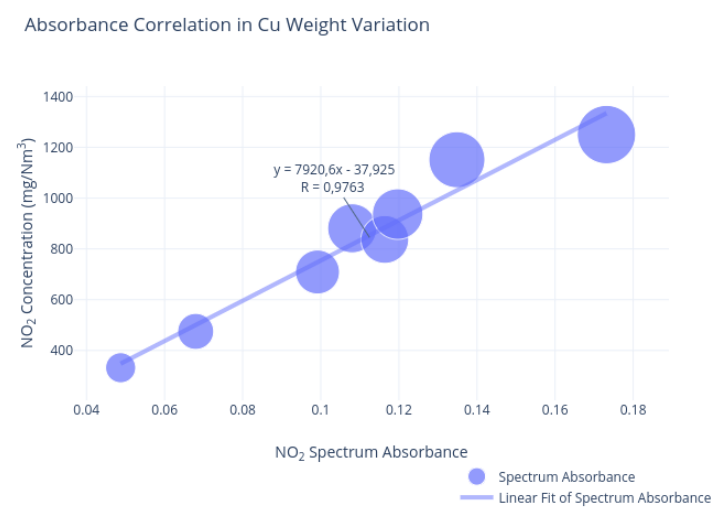

Figure 4. Absorbance Corelation in Copper Weight Variation

In other experiment, we made trial on Copper weight variation on fixed nitric acid molarity, this result more than $95 \%$ liniearity. Generated NO2 read by USEPA method 7B standard method using UV-Vis Spectrophotometer, while CCD Spectrometer read absorbance intensity. Absorbance intensity have linear correlation to copper weight and $\mathrm{NO} 2$ concentration.

In conclusion we have developed a new instrument setup for $\mathrm{NO} 2$ gas measurement using DOAS System correlated with USEPA Method 7 standard method. This measurement used $\mathrm{Cu}+\mathrm{HNO} 3$ reaction to generate NO2 gas. Entire measurement on DOAS System seems to be positive result for alternative method in $\mathrm{NO} 2$ measurement. We are in the process of developing this method and measurement for $\mathrm{NO} 2 \mathrm{gas}$ in industrial emission, combine with IoT Technology.

\section{Acknowledgments}

The author express their gratitude to Dr, Ali Murtopo Simbolon, ST, SSi, MM Head of the Center of Industrial Pollution Prevention Technology, Ministry of Industry, for providing necessary facilities.

\section{References}

Cross, Eben S., Leah R. Williams, David K. Lewis, Gregory R. Magoon, Timothy B. Onasch, Michael L. Kaminsky, Douglas R. Worsnop, and John T. Jayne. 2017. "Use of Electrochemical Sensors for Measurement of Air Pollution: Correcting Interference Response and Validating Measurements." Atmospheric Measurement Techniques 10 (9):3575-88. https://doi.org/10.5194/amt-10-3575-2017.

Ivanković, Tomislav, and Jasna Hrenović. 2010. "Surfactants in the Environment." Archives of Industrial Hygiene and Toxicology 61 (1):95-110. https://doi.org/10.2478/10004-1254-61-20101943.

Jörg Kleffmann, *, and Thorsten Benter, and Peter Wiesen. 2004. "Heterogeneous Reaction of Nitric Acid with Nitric Oxide on Glass Surfaces under Simulated Atmospheric Conditions." American Chemical Society . https://doi.org/10.1021/JP040184U.

Maeda, Yasuaki., Kenji. Aoki, and Makoto. Munemori. 1980. "Chemiluminescence Method for the Determination of Nitrogen Dioxide." Analytical Chemistry 52 (2). American Chemical Society:307-11. https://doi.org/10.1021/ac50052a022.

Naidoo, Rajen N. 2019. "NO2 Increases the Risk for Childhood Asthma: A Global Concern." The Lancet. Planetary Health 3 (4). Elsevier:e155-56. https://doi.org/10.1016/S25425196(19)30059-2.

Otten, C., F. Ferlemann, U. Platt, T. Wagner, and K. Pfeilsticker. 1998. "Groundbased Doas UV/Visible Measurements at Kiruna (Sweden) during the Sesame Winters 1993/94 and 1994/95." Journal of Atmospheric Chemistry 30 (1). Kluwer Academic Publishers:141-62. https://doi.org/10.1023/A:1005810732347. 
Platt, Ulrich, and Jochen Stutz. n.d. "DOAS: Yesterday, Today and Tomorrow." In Differential Optical Absorption Spectroscopy, 495-504. Berlin, Heidelberg: Springer Berlin Heidelberg. Accessed May 28, 2019. https://doi.org/10.1007/978-3-540-75776-4_12.

Stutz, Jochen, Bodo Werner, Max Spolaor, Lisa Scalone, James Festa, Catalina Tsai, Ross Cheung, et al. 2017. "A New Differential Optical Absorption Spectroscopy Instrument to Study Atmospheric Chemistry from a High-Altitude Unmanned Aircraft." Atmospheric Measurement Techniques 10 (3):1017-42. https://doi.org/10.5194/amt-10-1017-2017.

Zhang, Lu, Chengxi Yan, Qing Guo, Junbiao Zhang, and Jorge Ruiz-Menjivar. 2018. "The Impact of Agricultural Chemical Inputs on Environment: Global Evidence from Informetrics Analysis and Visualization." International Journal of Low-Carbon Technologies 13 (4). Narnia:338-52. https://doi.org/10.1093/ijlct/cty039.

Zhang, Renyi, Xuexi Tie, and Donald W Bond. 2003. "Impacts of Anthropogenic and Natural NO(x) Sources over the U.S. on Tropospheric Chemistry." Proceedings of the National Academy of Sciences of the United States of America 100 (4). National Academy of Sciences:1505-9. https://doi.org/10.1073/pnas.252763799.

Zielcke, Johannes, Martin Horbanski, Denis Pöhler, U Frieß, and Ulrich Platt. 2013. A New Calibration System for Lightweight, Compact and Mobile Cavity-Enhanced Differential Optical Absorption Spectroscopy Instruments. 